\title{
Factores pronósticos en la recidiva y progresión del cáncer superficial vesical. Grupos de riesgo (Parte I)
}

\author{
García Rodríguez J, Fernández Gómez JMª , Escaf Barmadah S, González Álvarez RC, \\ Rodríguez Robles L, Miranda Aranzubia O.
}

\author{
Servicio de Urología I. Hospital Central de Asturias. Departamento de Especialidades Médico-Quirúrgicas. \\ Facultad de Medicina. Universidad de Oviedo.
}

Actas Urol Esp. 2006;30(10):998-1008

\begin{abstract}
RESUMEN
FACTORES PRONÓSTICOS EN LA RECIDIVA Y PROGRESIÓN DEL CÁNCER SUPERFICIAL VESICAL. GRUPOS DE RIESGO (PARTE I)

Introducción: Hemos realizado un estudio retrospectivo sobre una serie de tumores superficiales vesicales, analizando los factores clínicopatológicos que pueden determinar la evolución posterior del tumor en cuanto a recurrencia y progresión.

Material y Métodos: Se revisaron en total 473 neoplasias vesicales superficiales, de las cuales finalmente 419 fueron útiles para el estudio (223 tumores primarios y 196 recurrentes). Se realizan estudios uni y multivariante sobre 24 variables de cada tumor referidas a las características y a la evolución de la neoplasia.

Resultados: Encontramos diferencias significativas en el tiempo libre hasta la recurrencia, que disminuyó en los tumores que previamente habían recidivado precozmente ( $<12$ meses), en los tumores múltiples ( 3 o más tumores) y los localizados en cúpula vesical. Además existieron diferencias estadísticamente significativas en el tiempo libre de enfermedad cuando se compararon dos grupos homogéneos de cirujanos mientras que el empleo de instilaciones vesicales incrementó dicho tiempo. En cuanto a tiempo hasta la progresión, éste disminuye en tumores que habían recurrido precozmente (en 6 meses), tumores mayores de $3 \mathrm{~cm}$, tumores de alto grado y según el grupo de cirujanos. Además, encontramos que la terapia de mantenimiento con BCG (bacilo Calmette-Guerin) demostró un mayor tiempo hasta la progresión frente al de inducción.

Discusión: Resultaron independientes para explicar menor tiempo libre hasta la recurrencia superficial los siguientes factores: recidiva en el $1^{\circ}$ año, multiplicidad, técnica quirúrgica, no empleo de lavados endovesicales, tratamiento con dosis bajas de BCG y terapia de inducción frente a mantenimiento. Respecto a la progresión, objetivamos que los factores con mayor influencia en la progresión fueron: recurrencia en los primeros 6 meses, grado (grado 2 y grado $3+$ Tis) y empleo de terapia endovesical de mantenimiento frente a inducción.

Palabras clave: Cáncer vesical. Superficial. Factores pronósticos.
\end{abstract}

\section{ABSTRACT \\ PRONOSTIC FACTORS ON RECURRENCE AND PROGRESSION OF SUPERFICIAL BLADDER CANCER. RISK GROUPS (PART I)}

Introduction: We have carried out a retrospective study on a series of superficial bladder tumours, analyzing the clinical-pathological factors that can determine the subsequent evolution of the tumour as for recurrence and progression.

Material and Methods: They were revised on the whole 473, of which finally 419 superficial bladder tumours were useful for the study (223 primary tumours and 196 recurrent). Studies are carried out univariate and multivariate on 24 variables of each tumour referred to the characteristics and to the evolution of the tumour.

Results: We find significant differences in the free time to recurrence, diminishing it in the tumours that recurred in less than 12 months, in multiple tumours (3 or more tumours) and in bladder dome tumours. Also statistically significant differences existed when were studied two homogeneous groups of surgeons, while the treatment with bladder instillations increased it. The time to progression, diminishes in: Tumours that had recurred prematurely (in 6 months), tumours over $3 \mathrm{~cm}$, high grade tumours and when two groups of surgeons were studied, we also find that the maintenance therapy with BCG (bacillus Calmette-Guerin) showed a greater time to progression versus induction therapy.

Discussion: The independent factors to explain smaller free time till superficial recurrence were: recurrence in the $1^{\circ}$ year, multiplicity, surgical technique, not employment of bladder washes, treatment with low dose of BCG and use of intravesical therapy with induction therapy versus maintenance. About the progression, we objectify that the factors with greater influence in the progression were, recurrence in the first 6 months, grade (grade 2 and grade $3+$ Tis) and treatment with maintenance therapy versus induction.

Key words: Bladder cancer. Superficial. Prognostic factors. 
$\mathrm{E}$ ntendemos por carcinoma superficial aquel limitado al epitelio vesical (Ta) o a la lámina propia (T1) de la pared vesical. Para la estadificación se utiliza habitualmente el sistema TNM desarrollado conjuntamente por la Unión Internacional Contra el Cáncer (UICC, International Union Against Cancer) y el American Commitee on Cancer Stating (AJC), del que la última revisión se ha realizado en el $2002^{1}$. Los tumores Ta representan un $70 \%$ de los superficiales y son generalmente de bajo o intermedio grado. Los T1 representan un $30 \% \mathrm{y}$, de estos últimos, aproximadamente un $10 \%$ son T1G3 (alto grado) en el momento del diagnóstico. La tendencia a la recidiva se cifra globalmente en torno a un $60-90 \% \%^{2}$ y la progresión en torno a un $10-30 \%$. Para las neoplasias Ta, la posibilidad de recidiva alcanza un $50 \%$ y para las $\mathrm{T} 1$ más del $70 \%$, siendo para la progresión de $<5 \%$ y del $30-50 \%$, respectivamente. Respecto al grado, los tumores G1-2 muestran recidivas cercanas al 50-60\% mientras que los de alto grado (G3) recidivan hasta en el $80 \%$ de los $\operatorname{casos}^{3}$. Asímismo los grados 1, 2, y 3 progresarán en un 2,11 y $45 \%$, respectivamente ${ }^{2}$.

Para predecir la evolución futura del tumor vesical superficial es imprescindible el conocimiento de factores pronósticos fiables. Se han publicado algunos estudios acerca de factores pronósticos del carcinoma superficial de vejiga ${ }^{4-8}$. Nosotros intentamos aportar un análisis riguroso de los factores que puedan estar implicados tanto en recurrencia, como en progresión, así como el peso de cada uno de ellos mediante el estudio de una amplia población de pacientes.

\section{MATERIAL Y MÉTODOS}

Se ha realizado un estudio retrospectivo sobre la base de datos de tumores vesicales corres- pondiente a pacientes tratados en el Servicio de Urología I del Hospital Universitario Central de Asturias, entre los años 1987 y 2002. Se revisaron en total 473 neoplasias vesicales superficiales, de las cuales finalmente 419 fueron útiles para el estudio (223 tumores primarios y 196 recurrentes). Se evaluó desde el punto de vista de cada tumor. Se recogieron 24 variables de cada neoplasia referidas a las características de estas y a la evolución en cuanto a recurrencia y progresión, éstas quedan recogidas en la Tabla 1.
Tabla 1

Variables

\section{Tipo}

№ de recidiva

Meses hasta recurrencia actual desde última recidiva

Tiempo total desde el diagnóstico

Tratamientos adyuvantes

previos

Sexo

Edad

Factores de riesgo

Tamaño del mayor tumor

№ tumores

Localización

Grado

$\mathrm{T}$ (estadio tumoral)

Cis

Lavados

Tipo

Cirujano

Recurrencia

Tiempo de recurrencia

Recurrencia en tracto urinario superior

Recurrencia en grado

Tiempo de seguimiento total

Número de recurrencias posteriores

Tiempo de seguimiento libre de enfermedad
Primario o una recidiva

№

Tiempo libre de enfermedad desde la recurrencia anterior

Tiempo desde el diagnóstico hasta la recidiva actual

$\mathrm{Si} / \mathrm{no}$

Varón/mujer

Tabaco/ocupacional/no

$>3 \mathrm{~cm} /<3 \mathrm{~cm}$

$>3 /<3$

Laterales/fondo-suelo/trígono/ Uretraprostaticacuello/cupula/ Papilomatosis

G1/G2/G3

$\mathrm{Ta} / \mathrm{T} 1 / \mathrm{NBPM}$

$\mathrm{Si} / \mathrm{no}$

Tratamiento endovesical, tipo y dosis

Inducción-Mantenimiento

Tumor recurrió o no y si recidiva fue superficial o profunda

Tiempo hasta la siguiente recurrencia

$\mathrm{Si} / \mathrm{no}$

Grado en que recurrió

Tiempo transcurrido desde el actual tumor primario/recurrente hasta el final del seguimiento

Número de veces que el tumor recurrió durante su seguimiento

Meses sin enfermedad hasta el final del seguimiento 
A partir del diagnóstico y la resección transuretral (RTU) vesical, y en función del resultado anatomopatológico, se plantearon distintos esquemas de seguimiento y tratamiento complementario. En general, en los tumores G3 y/o Tis (in situ), iniciamos tratamientos adyuvante utilizando dosis variables de BCG 81, 54, 27 y 13,5 mg con un ciclo inicial de 6 instilaciones y con programas de mantenimiento en algunos casos (3 instilaciones quincenales vesicales a los 3,6 y 9 meses). También se utilizó Mitomicina C ( MMC) 30 ó $40 \mathrm{mg}$ con programas de 6 instilaciones semanales, con o sin mantenimiento $(1 / 3$ meses hasta 3 años), en los casos en los que estuvo contraindicada la BCG.

La cepa de BCG que utilizamos fue la Connaught. Para controlar los efectos secundarios de la BCG endovesical, además de recogida de efectos adversos, se realizó Mantoux previo a tratamiento con BCG y en el primer control a los tres meses, momento en el que también se solicitó hemograma, bioquímica, cultivo BKs-Ziehl en orina, para seguir posteriormente a los pacientes con cultivo BKs-Ziehl en orina y Mantoux cada 6 meses. Tras 6 lavados realizamos BMN a los 3 meses en muchos casos y cuando se objetivó recidiva se realizó otro ciclo de 6 lavados y reevaluación bajo anestesia. En caso de persistencia de enfermedad de alto grado o invasión muscular se indicó cistectomía radical y derivación o sustitución urinaria.

En general, el seguimiento posterior a la RTU se planteó en estos casos cada 3-4 meses durante 2 años con cistoscopia y citologías, para continuar cada 6 meses durante el $3^{\circ}$ a $5^{\circ}$ año.

El análisis estadístico se realizó mediante el programa SPSS, versión 11. Se consideró la existencia de significación estadística cuando la $\mathrm{p}$ fue menor de 0,05. Las variables cuantitativas se expresaron como media \pm D.E (desviación estándar) o bien como mediana (percentil 50) en los casos pertinentes (tiempo de supervivencia). Se utilizó el test de Student para comparar las medias de dos categorías dentro de una misma variable dependiente y el análisis de la varianza (razón F) para comparar más de dos medias. Para conocer la existencia de asociación entre variables realizamos tablas de contingencia y la prueba de $\mathrm{Chi}^{2}$ de Pearson. El análisis de superviven- cia se realizó mediante el método de KaplanMeier, lo cual permitió su representación gráfica. Posteriormente, se realizó la prueba del rango logarítmico (log-rank) Por último, consideramos las variables que fueron significativas en el análisis univariante como covariantes en la prueba del modelo de riesgos proporcionales de Cox, con la intención de determinar el efecto conjunto de los factores pronósticos que resultan significativos de forma independiente y cuantificar los riesgos relativos ("odds ratio") e intervalos de confianza (IC) del 95\% de recurrencia.

\section{RESULTADOS}

Encontramos 223 tumores primarios y 196 recurrentes. Son 350 varones (83,5\%) y 69 mujeres (16,5\%). En cuanto a las características de los tumores, en 256 casos $(61,1 \%)$ se encontró un antecedente de tabaquismo (255 varones), en 4 (1\%) una posible influencia del ambiente laboral y en $159(37,9 \%)$ ningún factor. El tamaño medio del tumor encontrado fue significativamente mayor en varones que en mujeres. En cuanto al número de tumores, en un 19,3\% de casos hallamos tumores múltiples (>3). En 154 casos $(36,7 \%)$ el tumor era de grado I, en 175 casos $(41,8 \%)$ de grado II y en 83 pacientes $(19,8 \%)$ de grado III. Además, se encontraron 5 neoplasias de bajo poder de malignidad (NBPM) $(1,2 \%)$ y 2 Tis aislados $(0,5 \%)$. Hubo $48(11,4 \%)$ casos que tenían Tis asociado, encontrando asociación estadística entre la presencia de Tis y tumores de alto grado (GIII) cuando el carcinoma "in situ" se asoció a tumores exofíticos. En cuanto al estadio, además de la NBPM y los 2 casos de Tis aislados, se encontraron 70 pacientes $(16,7 \%)$ con tumores Ta y $342(81,6 \%)$ con tumores T1. En 219 casos $(52,3 \%)$ se empleó un tratamiento endovesical tras la RTU.

El tiempo de seguimiento medio global fue de $39,95 \pm 32,14$ meses (3-151 meses) con una mediana de 30 meses. Encontramos recurrencia tumoral en 223 pacientes $(53,2 \%)$, de los cuales la recidiva fue superficial en 182 casos $(81,6 \%)$ y con invasión del detrusor (lo que denominaremos progresión) en $41(18,4 \%)$, lo cual supone que globalmente hubo crecimiento en profundidad (T2-4) en el 9,8\% de casos de nuestra serie. 
Es importante explicar que en esta serie se estudia cada tumor, primario o recurrente por separado. El número de recurrencias previas, es decir el número de veces que cada tumor había recurrido antes (sin contar el primario del estudio) fue de $2,1 \pm 1,67$ (1-11) y el tiempo de recurrencia previo fue de 20,3 $\pm 22,03(2-120)$ meses. La mediana del tiempo de seguimiento tras cada RTU fue de 30 meses. En los tumores que recurrieron, el tiempo libre de enfermedad hasta recurrencia superficial, es decir, el tiempo que tarda en recidivar el tumor desde el momento actual, fue de 24,87 $\pm 24,5$ meses (3-120 meses), con una mediana de 12 meses (Tabla 2). La media del tiempo libre de enfermedad en los pacientes que presentaron progresión fue de $16,5 \pm 14,10$ meses (3-72 meses), con una mediana de 12 meses. Respecto a la recurrencia según estadio y grado podemos observar los resultados en la Tabla 3A y 3B.

\section{Factores clínico-patológicos}

\section{Recurrencia}

En el análisis univariante, encontramos en los tumores recurrentes, una asociación significativa entre sexo y tiempo de recurrencia previo, siendo 1,8 veces mayor el riesgo en mujeres con tumores recurrentes respecto a los varones de padecer estas neoplasias en menos de 6 meses de la anterior. También entre multiplicidad y sexo, de forma que las mujeres tuvieron un riesgo relativo mayor de tener tumores múltiples que los varones.

Los tumores recurrentes tendieron a ser múltiples y menores de $3 \mathrm{~cm}$, comparados con los primarios, que presentaron mayor proporción de tumores de $\geq 3 \mathrm{~cm}$ y únicos (1 ó 2 tumores). Respecto a la localización, los tumores de cúpula tuvieron una probabilidad 2,9 veces mayor de ser recurrentes.

Tabla 2

Estadísticos descriptivos de la serie. Datos cuantitativos

\begin{tabular}{|c|c|c|c|c|c|}
\hline & $\mathbf{N}$ & Mínimo & Máximo & Media & Desv. tipica \\
\hline № de recidivas (recurrentes) & 196 & 1 & 11 & 2 & 1,6 \\
\hline Meses desde tumor previo (recurrentes) & 196 & 2 & 120 & 20,3 & 22 \\
\hline Edad (años) & 419 & 39 & 90 & 69,3 & 10,1 \\
\hline Tamaño (cm) & 419 & 0,5 & 6 & 1,5 & 1,1 \\
\hline Número de tumores & 419 & 1 & 30 & 2,3 & 2,4 \\
\hline Tiempo de seguimiento total (meses) & 419 & 3 & 151 & 39,9 & 32,1 \\
\hline Tiempo libre de enfermedad (meses) & 419 & 3 & 120 & 24,8 & 24,5 \\
\hline
\end{tabular}

Tabla 3A

Distribución del número de tumor que resulta tras la recurrencia en relación al grado del tumor del que recurre.

\begin{tabular}{|c|c|c|c|c|}
\hline \multirow[b]{2}{*}{ Grado del tumor que recurre } & \multicolumn{3}{|c|}{ Grado del tumor en el que recurre } & \multirow[b]{2}{*}{ Total } \\
\hline & G1 & G2 & G3-Tis & \\
\hline GI & 51 & 20 & 7 & 78 \\
\hline GII & 32 & 44 & 24 & 100 \\
\hline GIII-Tis & 5 & 9 & 29 & 43 \\
\hline Total 88 & 73 & 60 & 221 & \\
\hline
\end{tabular}

Tabla 3B

Distribución del número de tumor que resulta tras la recurrencia en relación al estadio del tumor que resulta.

\begin{tabular}{cccc}
\hline Estadio del tumor en el que recurre & \multicolumn{2}{c}{ Grado del tumor en el que recurre } \\
& G1 & G2 & 65 \\
Ta-T1-Tis & 88 & 65 & 27 \\
T2-T4 & 0 & 83 & 41 \\
Total 88 & 73 & 60 & 221 \\
\hline
\end{tabular}


Encontramos una asociación significativa $\left(\mathrm{Chi}^{2}\right)$ entre tipo (primario/recurrente) y grado tumoral, de forma que los tumores de alto grado (grado III) fueron con mayor frecuencia primarios. También el tamaño medio se incrementó significativamente con el grado.

Existió mayor frecuencia de tumores T1 primarios $(58,2 \%$ de $\mathrm{T} 1)$ respecto a los $\mathrm{Ta}$, siendo el tamaño tumoral medio significativamente mayor en los tumores T1 frente a los Ta.

Estudiamos ahora el tiempo hasta la recurrencia analizando la influencia de posibles factores de riesgo y encontramos diferencias significativas respecto a este, es decir, un menor tiempo hasta la recurrencia en las siguientes variables. Tumores que habían recurrido en menos de 12 meses, tumores múltiples (3 o más tumores) y tumores de cúpula.

Para conocer la posible influencia de distintos cirujanos en la evolución posterior del tumor, teniéndose en cuenta que se trata de una serie retrospectiva, se estudiaron primero las curvas de recurrencia de cada urólogo incluido en la serie observando en estas diferencias significativas. A partir de este dato creamos dos grupos homogéneos, es decir, sin diferencias en las características de los tumores operados, donde volvemos a observar estas diferencias en el tiempo hasta recurrencia (esta variable la definiremos como técnica quirúrgica).

Nos parece importante reseñar que respecto al estadio y grado no encontramos diferencias significativas (p: 0,9076), asimismo la presencia de carcinoma in situ aislado o asociado tampoco mostró diferencias estadísticamente significativas en cuanto al tiempo libre de enfermedad.

\section{Progresión}

En cuanto a progresión, obtenemos que existen diferencias significativas en el tiempo hasta la progresión, disminuyéndolo, en tumores que habian recurrido precozmente (en 6 meses), tumores mayores de $3 \mathrm{~cm}$, y según la técnica quirúrgica.

Cuando se estudió el grado de malignidad, encontramos diferencias significativas $(\mathrm{p}<0,001)$ en el tiempo de progresión, siendo este tiempo para los tumores de grado 1 mayor que para los de grado 2 y éste, a su vez, mayor que el de los tumores de grado 3 (Fig. 1). La presencia de carcinoma in situ aislado o asociado no mostró diferencias estadísticamente significativas en cuanto al tiempo hasta la progresión, si bien las diferencias se aproximaron a la significación estadística (p: 0,0595). En el resto de variables no hallamos significación estadística.

\section{Tratamiento}

Respecto al tratamiento endovesical sabemos que el empleo de instilaciones vesicales incrementó el tiempo hasta la recurrencia (Fig. 2).

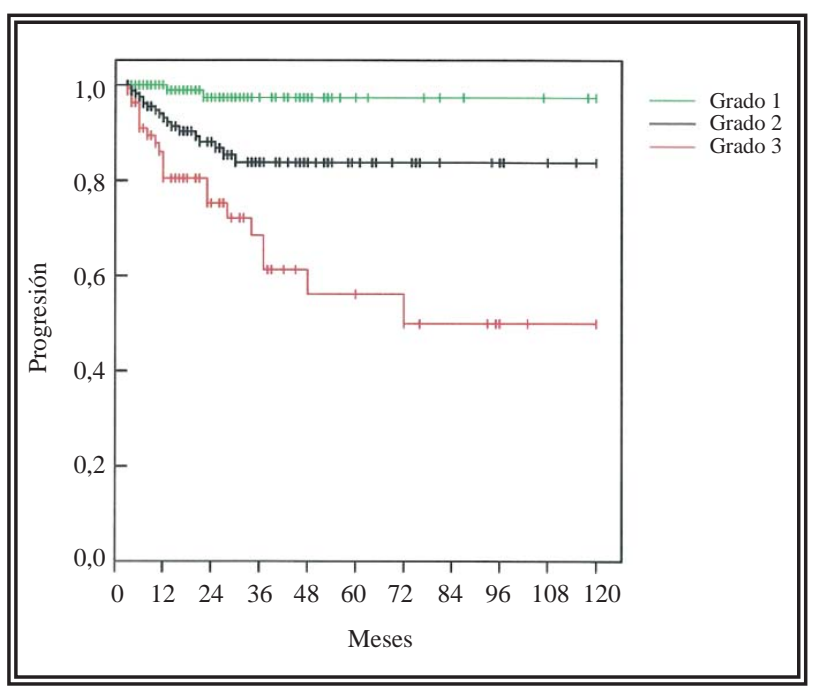

FIGURA 1. Tiempo hasta la progresión según el grado (excluyendo Tis y NBPM), mostrando diferencias significativas ( $p<0,001)$.

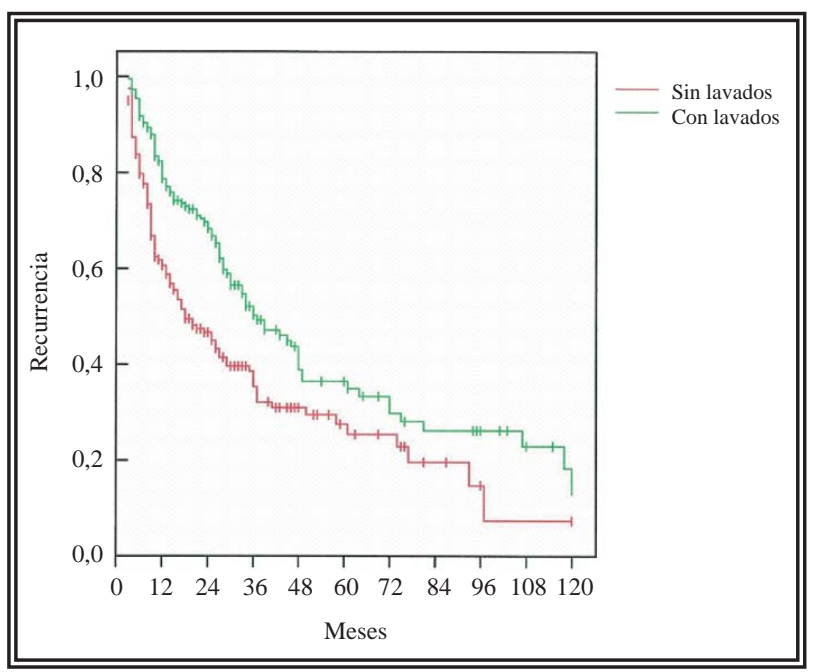

FIGURA 2. Tiempo hasta la recurrencia según se emplearan o no lavados vesicales tras la RTU, mostrando diferencias significativas (p:0,0001). 
La mediana del tiempo libre de enfermedad en los pacientes que no recibieron tratamiento fue de 18 meses y el de los que recibieron tratamiento fue de 37 meses.

Además se demostró que existían diferencias significativas al emplear BCG o quimioterapia con diferentes drogas, fundamentalmente MMC. La mediana en los que fueron tratados con BCG fue de 75 meses, la de los pacientes que habían recibido quimioterapia endovesical fue de 45 meses y la de los que no recibieron tratamiento fue de 25 meses.

La terapia de mantenimiento demostró un mayor tiempo libre de enfermedad comprobándose que existían diferencias significativas en el tiempo hasta la recurrencia (p: 0,0292), con un tiempo mayor en los pacientes que habian recibido dosis altas de BCG. Reagrupamos en dos grupos para el estudio multivariante, uno de ellos el de BCG a dosis altas y el otro una combinación de tratamiento con BCG de baja dosis quimioterapia o nada, comprobando nuevamente que existían diferencias estadísticamente significativas $(p<0,001)$ en el tiempo medio libre de enfermedad a favor del grupo de dosis altas de BCG.

En cuanto al tiempo de progresión, globalmente, el empleo o no de instilaciones vesicales no mostró diferencias estadísticamente significativas (p: 0,0614). La terapia de mantenimiento (más de 6 lavados) demostró un mayor tiempo hasta la progresión frente al de inducción (6 lavados semanales). La terapia de mantenimiento, curiosamente mostró un tiempo hasta la progresión similar a la de los pacientes que no habían recibido tratamiento endovesical, esto se debe a que el grupo sin tratamiento corresponde a casos de tumores de bajo grado, con mejor pronóstico (Fig. 3).

Finalmente, aplicando el modelo de riesgos de Cox resultaron independientes para explicar menor tiempo libre hasta la recurrencia superficial los siguientes factores: recidiva en el $1^{\circ}$ año, multiplicidad, técnica quirúrgica, no empleo de lavados endovesicales, tratamiento con dosis bajas de BCG y terapia de inducción frente a mantenimiento, mientras que para la progresión resultaron independientes: recurrencia en los primeros 6 meses, grado (grado 2 y grado $3+$ Tis) y empleo de terapia endovesical con terapia de

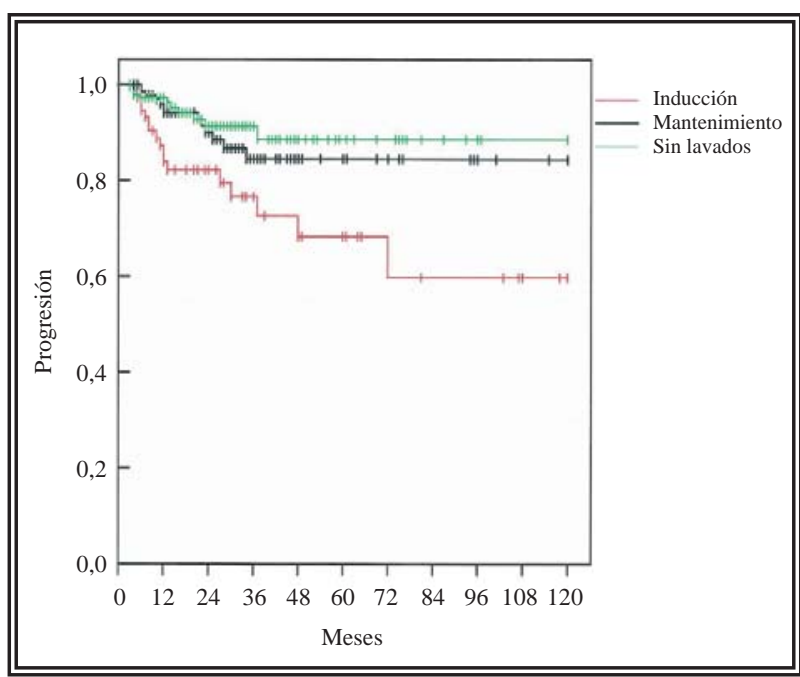

FIGURA 3. Tiempo hasta la progresión según la duración de la terapia endovesical (vs no tratamiento), mostrando diferencias significativas (p:0,046).

inducción frente a mantenimiento. Basándonos en los Riesgos Relativos u OR observamos como factor claramente protector para recurrencia el uso de BCG con una $\mathrm{OR}<1$ (dosis altas de BCG disminuyen el riesgo de recurrencia, $O R<1$ ), y el resto de factores lo aumentan $(\mathrm{OR}>1)$ (Tabla 4), mientras que en cuanto a progresión el Grado III con una OR de 20,470 resulta un factor claramente agresivo (Tabla 5).

\section{DISCUSIÓN}

El cáncer de vejiga tiene un origen multifactorial. El problema básico es aclarar la patogenia de la enfermedad, lo cual resulta fundamental para descubrir los factores determinantes de su recurrencia y progresión. En relación con esto último, el planteamiento general que nos hicimos al comenzar este trabajo estuvo basado en intentar conocer los factores clínicopatológicos que podían determinar el mayor o menor riesgo para la evolución posterior del tumor.

En los últimos años, se ha producido un notable esfuerzo en el estudio de los factores etiológicos y pronósticos del cáncer de vejiga, dada su prevalencia y el constatado aumento de su incidencia (hasta un 50\% más desde la década de los años 50).

Oosterlinck, en el año 2004, hace una revisión de las líneas guía de la Asociación Europea de Urología (EAU) donde analiza los factores con 


\section{Tabla 4}

Variables independientes para la recurrencia en el modelo de riesgo proporcional de Cox, con los odds ratio correspondientes y su representación gráfica (dosis altas de BCG disminuyen el riesgo de recurrencia, $\mathrm{OR}<1$, y el resto de factores lo aumentan $(\mathrm{OR}>1)$

\begin{tabular}{lc}
\hline & (OR) \\
\hline Inducción & 1,623 \\
Sin lavados & 2,218 \\
& \\
Dosis altas de BCG & 0,4694 \\
& \\
Técnica quirúrgica & 1,507 \\
Multiplicidad & 2,160 \\
Meses de recurrencia previa & 1,505 \\
\hline
\end{tabular}

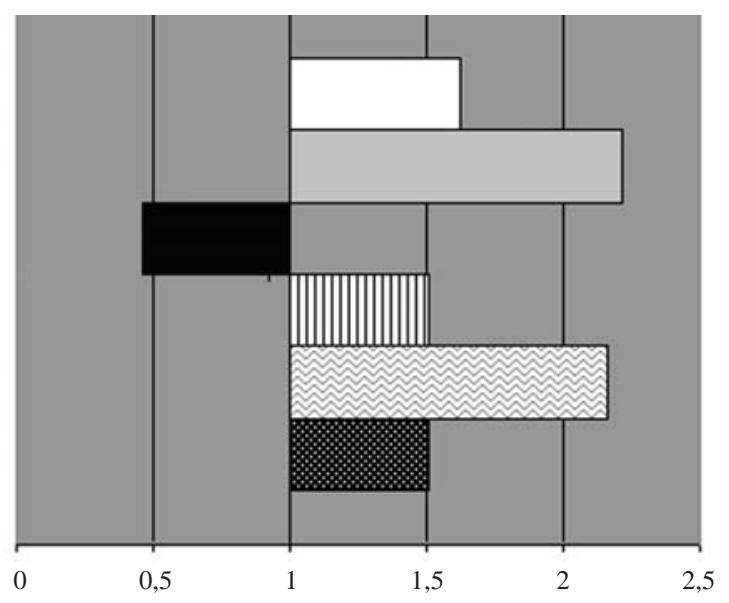
$\square$ Inducción (respecto a mantenimiento)
$\square$ Sin lavados (respecto a mantenimiento)
Dosis altas (BCG)
㿼 Técnica quirúrgica (Grupo A vs Grupo B)
图 Multiplicidad (Tumores múltiples)
圈 Meses de recurrencia previos (<12 meses)

mayor peso en la recidiva ${ }^{9}$. En primer lugar destaca la multifocalidad, es decir, el número de tumores, observando que los pacientes con más tumores tenían más frecuentemente recidivas, y si existían más de 6 tumores, la recurrencia llegaba prácticamente al 100\%. En segundo lugar el factor con mayor peso fue la tasa de recurrencia previa, calculada por la recurrencia a los 3 meses postRTU. Nuestros resultados coinciden, ya que la multiplicidad resultó en nuestros pacientes ser un factor favorecedor de recurrencia, con un menor tiempo libre de enfermedad cuando había 3 o más tumores. Igualmente ocurrió con el tamaño, puesto que por encima de los $3 \mathrm{~cm}$ los tumores presentan mayor tasa de recurrencia. Igualmente, para Millán-Rodríguez et al. la multiplicidad tumoral y el tamaño por encima de 3 cm conllevan un incremento en el riesgo de recurrencia $^{10}$.

Respecto a recurrencia temprana, en nuestra serie el 19\% de los tumores que recurrieron lo hicieron en menos de 6 meses desde la RTU. Encontramos en el estudio multivariante que la recidiva en los 6 primeros meses fue un factor independiente que influyó en el tiempo hasta la recidiva superficial. A esta misma conclusión se llega también en un trabajo realizado por Ali-ElDein et al., en el que la recurrencia en la primera cistoscopia a los 3 meses, junto al grado histológico y la ploidia del $\mathrm{ADN}$, son los únicos predictores de progresión del tumor ${ }^{11}$. Asímismo

\section{Tabla 5}

Variables independientes para la progresión en el modelo de riesgo proporcional de Cox, con los odds ratio correspondientes y su representación gráfica.

\begin{tabular}{lc}
\hline & $\begin{array}{c}\text { Odds } \\
\text { ratio }\end{array}$ \\
\hline Inducción (respecto a mantenimiento) & 2,557 \\
Sin lavados (respecto a mantenimiento) & 1,715 \\
Meses de recurrencia previa (> 6 meses) & 3,858 \\
Grado 2 (respecto a grado 1) & 6,852 \\
Grado 3 (respecto a grado 1) & 20,470
\end{tabular}

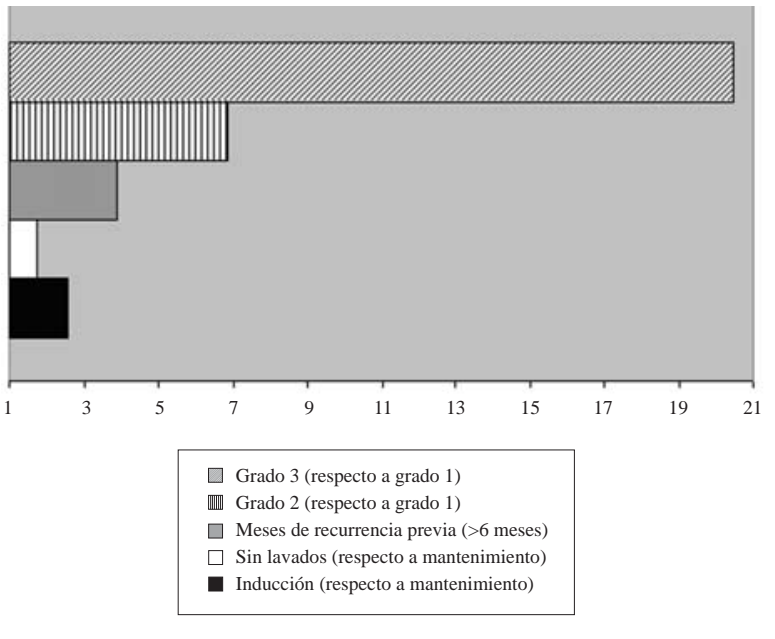


para la Asociación Europea de Urología la alta frecuencia de recurrencias implica que el tumor se incluya dentro de los de alto riesgo ${ }^{9,12}$. Respecto a este punto, en una revisión de siete estudios en fase III de la EORTC se encontró una gran variabilidad en cuanto a la recidiva en la primera cistoscopia de control a los tres meses en distintos centros, concluyendo la posibilidad de que la calidad de la resección realizada por distintos cirujanos podría explicar estas distintas tasas de recurrencia ${ }^{13}$. En nuestro estudio hemos analizado, para las mismas características tumorales, distintas técnicas quirúrgicas, teniendo siempre en cuenta que se trata de un hospital docente, obteniendo diferencias significativas en los tiempos libres de enfermedad, lo que corrobora estudios previos.

En cuanto a la localización, los tumores en cúpula vesical tienen en nuestro trabajo 2,9 veces más probabilidad de recurrir que los tumores de otras localizaciones. Tenemos que decir, además, que esta localización se asoció en nuestros resultados a multiplicidad, lo que incrementa el riesgo de recurrencia. Quizás nuestros resultados se relacionan con las dificultades técnicas que pueda ocasionar la resección de los tumores en dicha localización y/o probablemente a otros factores, como la mencionada multiplicidad asociada.

Respecto al grado de malignidad, los tumores de alto grado (G3) tienen una tendencia a la recurrencia de hasta el $80 \%{ }^{14}$, aunque en nuestro estudio el grado no resulta un factor influyente en el tiempo hasta la recurrencia superficial, ni siquiera cuando se considera el carcinoma insitu aislado o asociado. Millán-Rodríguez et al. analizan las variables implicadas en la recurrencia de 1.529 pacientes con carcinomas superficiales, concluyendo que la multiplicidad, el tamaño (mayor de $3 \mathrm{~cm}$ ), la presencia de Tis y el empleo de lavados intravesicales tras la RTU (disminuyendo la tasa de recurrencia en caso de su utilización), eran los factores más importantes ${ }^{10}$, entre estos no se incluye el grado.

$\mathrm{El}$ estadio tumoral en los tumores superficiales (Ta y T1), representa otra variable pronóstica importante en diversos estudios de carcinoma superficial. Encontramos mayor frecuencia de tumores $\mathrm{T} 1$ primarios respecto a los Ta, que ten- dieron a ser más frecuentemente recurrentes (71,4\% de Ta). Respecto a la recurrencia, una revisión sobre 2.410 pacientes Ta y $\mathrm{T} 1$ procedentes de 7 estudios en fase III de la EORTC mostraba una tendencia a la recidiva en la primera cistoscopia realizada tras la RTU vesical de un 3,4\% a un $45,8 \%^{13}$. Es decir, el estadio o la profundidad de la invasión tumoral en la pared de la vejiga parece marcar una diferencia en cuanto a pronóstico en diversas series, si bien, no todos los autores están de acuerdo en este punto, como por ejemplo Millán ${ }^{8}$. En nuestro estudio no hallamos diferencias significativas en el tiempo libre de enfermedad cuando tenemos en cuenta el estadio tumoral $\mathrm{T} 1$ frente a Ta.

$\mathrm{El}$ aspecto más importante a la hora de analizar la evolución del carcinoma vesical superficial es su tendencia a la progresión, por lo que implica en cuanto a la supervivencia de los pacientes. La progresión tumoral, marca claramente el pronóstico de un tumor y, en consecuencia, el del paciente. Sabíamos que de los pacientes con tumores G3 la mitad sufre progresión de la enfermedad, lo cual se traduce en tasas de supervivencia libre de progresión a 15 años de $61 \%$ y $44 \%$ para TaG3 y T1G3 respectivamente, y tasas de supervivencia específica para la enfermedad de $74 \%$ y $62 \%{ }^{14}$.

En distintos estudios se ha concluido que la proporción de progresiones es muy baja en los tumores grado 1, pero como decimos llega hasta el 50\% en los tumores T1G3 y Tis. En definitiva, se objetiva que el factor más importante en cuanto a progresión es el grado de anaplasia y en segundo lugar el estadio o categoría $\mathrm{T}^{2,15,16}$. Hemos visto a través del estudio realizado por el National Bladder Cancer Collaborative Group sobre 249 pacientes Ta y T1 tratados únicamente mediante resección transuretral, progresiones para Ta, T1 y para Grado 1 y 2 , y grado 3 de un 4, 30, 2,11 y 45\%, respectivamente ${ }^{2}$.

Para Millán-Rodríguez el principal predictor de progresión y mortalidad es el alto grado o G3 ${ }^{10}$. El grado 3, respecto al 1, el que presenta una odds ratio mayor y con una gran diferencia respecto a los demás factores, 19,9 para G3 frente a 3 para G2, respectivamente. Hemos encontrado diferencias significativas en el tiempo de progresión según el grado, siendo este tiempo 
para los tumores de grado 1 mayor que para los de grado 2 y éste, a su vez, mayor que el de los tumores de grado 3. Agrupando los tumores de alto grado con los pacientes que presentaban carcinoma in situ aislado o asociado a un tumor papilar (debido a su carácter agresivo), nuevamente encontramos diferencias significativas al estudiar el tiempo hasta la progresión, que fue mucho menor en los pacientes con tumores grado 3 y Tis aislado o asociado. Así Kwak evalúa sobre 75 pacientes tratados por tumores T1 G3 el riesgo de recurrencia y progresión según se trate de G3 primario o no primario, con un seguimiento medio de 100 meses. Concluyen un mayor potencial para recurrencia y progresión en el caso de tratarse de un tumor primario de alto $\operatorname{grado}^{17}$.

Asímismo, objetivamos que la recurrencia precoz, en los primeros 6 meses, es un factor de riesgo para la progresión, coincidiendo con el trabajo de Ali-El-Dein et al., ya comentado ${ }^{11}$.

$\mathrm{El}$ empleo de terapia endovesical como tratamiento adyuvante está ampliamente descrito en la literatura. Se han publicado diversos estudios y metaanálisis a este respecto, pero destacamos el realizado sobre 1.476 pacientes por la EORTC, donde se compara RTU sola frente a RTU más una instilación inmediata de un quimioterápico endovesical. Concluye que con la resección sola se producía una recurrencia del $48,4 \%$ frente a un $36,7 \%$ en los pacientes que recibían una instilación posterior a la cirugía ${ }^{18}$.

Comprobamos claramente en nuestro estudio que el empleo de instilaciones vesicales incrementó el tiempo hasta la recurrencia, con medianas de tiempo libre de enfermedad de 18 a 37 meses sin o con tratamiento, respectivamente, y que además, existían diferencias significativas al emplear BCG o quimioterapia con diferentes drogas, fundamentalmente MMC, con clara ventaja para la BCG. Según nuestros resultados, la mediana en los que fueron tratados con BCG fue de 75 meses, la de los pacientes que habían recibido quimioterapia endovesical fue de 45 meses y la de los que no recibieron tratamiento fue de 25 meses. A estas mismas conclusiones llegan otros estudios, como el realizado por Bohle, demostrando una superioridad estadísticamente significativa a favor de la BCG con terapia de manteni- miento para la prevención de la progresión y recurrencia frente a la $\mathrm{MMC}^{19,20}$.

En este sentido, la terapia de mantenimiento demostró un mayor tiempo libre de enfermedad, con una mediana de 46 meses, frente al de inducción con una mediana de 28 meses. A esta conclusión se llega igualmente en distintos estudios, como el publicado por Kolodziej et al. sobre 155 pacientes con tumores de alto riesgo que se randomizaron en RTU sola frente a RTU más terapia de mantenimiento con BCG, objetivando progresiones en el $23 \%$ frente al $8 \%$ de los pacientes $^{21}$.

El empleo de dosis altas ha sido un tema controvertido. El grupo CUETO ha puesto en marcha varios protocolos como el 90008, donde se ha comparado la dosis reducida de $27 \mathrm{mg}$ frente a la estándar de $81 \mathrm{mg}$ en tumores de riesgo intermedio y alto, encontrando diferencias estadísticamente significativas a 40,5 meses de seguimiento en la tasa de recidiva de los tumores múltiples, siendo de $22,7 \%$ en el brazo de los $81 \mathrm{mg}$ frente a un $40,8 \%$ en el brazo de $27 \mathrm{mg}$. También se objetivan diferencias en la tasa de progresión de Tis o primarios asociados a Tis, siendo del 11,9\% en el brazo de $81 \mathrm{mg}$ frente al 30,3\% en el brazo de $27 \mathrm{mg}^{22}$. En el año 2002, publican nuevos resultados comparando la dosis reducida $(27 \mathrm{mg})$ frente a la dosis estándar de $81 \mathrm{mg}$, determinando que la dosis reducida tiene similares resultados para recurrencia y progresión pero con una menor toxicidad, aunque recomiendan continuar con la dosis estándar de $81 \mathrm{mg}$ en los pacientes con tumores de alto riesgo ${ }^{23}$. Encontramos que el empleo de altas dosis de BCG produjo un tiempo mayor hasta la recurrencia frente a dosis bajas o el empleo de quimioterapia, incluso agrupando, para el estudio multivariante, los tratamientos con BCG a dosis altas en un grupo y en el otro una combinación de tratamiento con $\mathrm{BCG}$ de baja dosis, quimioterapia o nada, comprobamos nuevamente que existían diferencias significativas en el tiempo medio libre de enfermedad a favor del grupo de dosis altas de BCG.

Numerosos estudios confirman los beneficios del tratamiento adyuvante tras la RTU para lograr conseguir retrasar la progresión ${ }^{24,25}$. Un estudio de Peyromaure recientemente publicado, analiza 74 pacientes tratados por T1 G3. De estos 
57 son tratados mediante RTU y terapia adyuvante con BCG en régimen de mantenimiento, 10 mediante RTU y MMC y 7 pacientes mediante RTU solamente. Demuestran que en los pacientes del primer grupo la tasa de progresión fue del $23 \%$, significativamente inferior al resto de los grupos ${ }^{26}$. A las mismas conclusiones llega otro estudio de Shahin et al. sobre 153 pacientes diagnosticados de T1G3 que objetivan progresiones en el 33\% de pacientes tratados con RTU más terapia con BCG, frente a un $36 \%$ de progresión en pacientes tratados mediante RTU solamente ${ }^{27}$.

Nuestros resultados demuestran que existen diferencias estadísticamente significativas en el tiempo hasta la progresión al comparar tratamientos de inducción con BCG (6 lavados semanales) frente a mantenimiento (más de 6 lavados), con un claro beneficio a favor de la terapia de mantenimiento en demostrar un mayor tiempo hasta la progresión. Estos resultados coinciden con la mayoría de los trabajos publicados, como el metaanálisis, ya comentado, que se realizó sobre 4.863 pacientes comparando la RTU sola, RTU con tratamiento complementario con BCG y RTU con otros tratamientos distintos de BCG. Se concluyó en este estudio, que la terapia con BCG reduce significativamente el riesgo de progresión después de RTU en pacientes con carcinoma superficial vesical que reciben terapia de mantenimiento, sobre todo en pacientes con riesgo intermedio y alto y aquellos con Tis asociado, traduciéndose en una disminución del $27 \%$ en las tasas de progresión ${ }^{28}$.

\section{CONCLUSIONES}

Llegamos a las siguiente conclusiones:

A. Factores que influyeron de forma independiente en el tiempo hasta la recurrencia superficial:

\section{Disminuyéndolo}

1a. Recidiva previa durante el $1^{\mathrm{er}}$ año. 1b. Multiplicidad (3 o más tumores).

2. Una técnica quirúrgica apropiada y el empleo de instilaciones vesicales incrementaron de forma independiente el tiempo hasta la recidiva. La recurrencia se produjo significativamente más tarde cuando se habían empleado dosis altas de BCG y terapia de mantenimiento.
B. Factores que influyeron de forma independiente en el tiempo hasta la progresión:

1. Disminuyéndolo.

1a. Incremento del grado de malignidad.

1b. Recurrencia precoz, menor a 6 meses.

2. La utilización de terapia endovesical de mantenimiento implicó una progresión más tardía.

\section{REFERENCIAS}

1. Sobin LM, Witteking Ch. TNM Classification of malignant tumors. $6^{\text {a }}$ Edición Wiley-Liss. Nueva York. 2002;99205.

2. Heney NM, Ahmed S, Flanagan MJ, Frable W, Corder MP, Hafermann MD et al. Superficial bladder cancer: progression and recurrence. J Urol. 1983;130(6):1083-1086.

3. Lee R, Droller MJ. The natural history of bladder cancer. Implications for therapy. Urol Clin North Am. 2000;27(1): $1-13$.

4. Pagano F, Garbeglio A, Milani C, Bassi P, Pegoraro V. Prognosis of bladder cancer. I. Risk factors in superficial transitional cell carcinoma. Eur Urol. 1987;13(3):145-149.

5. Fujii Y, Fukui I, Kihara K, Tsujii T, Kageyama Y, Oshima H. Late recurrente and progresión alter a long tumor-free period in prymary Ta and T1 bladder cancer. Eur Urol. 1999;36(4):309-313.

6. Kiemeney LA, Witjes JA, Heijbroek RP, Verbeek AL, Debruyne FM. Predictability of recurrent and progressive disease in individual patients with primary superficial bladder cancer. J Urol. 1993;150(1):60-64.

7. Ali-El-Dein B, Sarhan O; Hinev A, Ibrahiem el-HI, Nabeeh A, Ghoneim MA. Superficial bladder tumours: analysis of prognostic factors and construction of a predictive index. BJU Int. 2003;92(4):393-399.

8. Millán-Rodríguez F, Chechile-Toniolo G, Salvador-Bayarri J, Palou J, Algaba F, Vicente-Rodríguez J. Primary superficial bladder cancer risk groups according to progression, mortality and recurrence. J Urol. 2000;164(3):680-684.

9. Oosterlinck W. Guidelines on diagnosis and treatment of superficial bladder cancer. Minerva Urol Nefrol. 2004,56(1): 65-72.

10. Millan-Rodriguez F, Chechile-Toniolo G, Salvador-Bayarri J, Palou J, Vicente-Rodriguez J. Multivariate analysis of the prognostic factors of primary superficial bladder cancer. J Urol. 2000;163(1):73-78.

11. Ali-El-Dein B, Sarhan O; Hinev A, Ibrahiem el-HI, Nabeeh A, Ghoneim MA. Superficial bladder tumours: analysis of prognostic factors and construction of a predictive index. BJU Int. 2003;92(4):393-399.

12. Oosterlinck W, Lobel B, Jakse G, Malmström PU, Stöckle M, Sternberg C. Guidelines on bladder cancer. Eur Urol. 2002;41(2):105-112.

13. Brausi M, Collette L, Kurth K, van der Meijden AP, Oosterlink W, Witjes JA et al. EORTC Genito-Urinary Tract Cancer Collaborative Group: Variability in the recurrence rate at first follow-up cystoscopy after TUR in stage Ta T1 transitional Cell carcinoma of the bladder: a combined analysis of seven EORTC studies. Eur Urol. 2002;41(5):523-531.

14. Herr HW. Tumor progression and survival of patients with high grade, noninvasive papillary (TaG3) bladder tumors: 15-year outcome. J Urol. 2000;163(1):60-61. 
15. Kurth KH, Denis L, Bouffioux C, Sylvester R, Debruyne FM, Pavone-Macaluso $\mathrm{M}$ et al. Factors affecting recurrence and progression in superficial bladder tumors. Eur J Cancer. 1995;31A(11):1840-1846.

16. Chen SS, Chen KK, Lin AT, Chang YH, Wu HH, Hsu TH et al. The significance of tumor grade in predicting disease progression in stage Ta transitional cell carcinoma of the urinary bladder. Br J Urol. 1996;78(2):209-212.

17. Kwak C, Ku JH, Park JY, Lee E, Lee SE, Lee C. Initial tumor stage and grade as a predictive factor for recurrence in patients with stage T1 grade 3 bladder cancer. J Urol. 2004;171(1):149-152.

18. Sylvester RJ, Oosterlinck W, van der Meijden AP. A single immediate postoperative instillation of chemotherapy decreases the risk of recurrence in patients with stage Ta T1 bladder cancer: a meta-analysis of published-results of randomised clinical trials. J Urol. 2004;171(6 Pt1):21862190.

19. Bohle A, Bock PR. Intravesical bacille Calmette-Guerin versus mitomycin $\mathrm{C}$ in superficial bladder cancer: formal meta-analysis of comparative studies on tumor progression. Urology 2004;63(4):682-686.

20. Bohle A, Jocham D, Bock PR. Intravesical bacillus Calmette-Guerin versus mitomycin $\mathrm{C}$ for superficial bladder cancer: a formal meta-analysis of comparative studies on recurrence and toxicity. J Urol. 2003;169(1):90-95.

21. Kolodziej A, Dembowski J, Zdorojowy R. Treatment of high-risk superficial bladder cancer with maintenance bacilli Calmette-Guerin therapy: preliminary results. BJU Int 2002;89(6):620-622.

22. Martínez-Piñeiro JA, Flores N, Isorna S et al. Estudio prospectivo comparando $81 \mathrm{mg}$ de BCG intravesical versus 27 mg de BCG en cáncer superficial de vejiga: Análisis definitivo del estudio CUETO 90008. XXIV Congreso de la Confederación Americana de Urología. Cancún 6-12 Nov. 1998. Libro de resúmenes. Abstr. TL 95.
23. Martínez-Piñeiro JA, Flores N, Isorna S, Solsona E, Sebastián JL, Pertusa C et al for CUETO (Club Urológico Español de Tratamiento Oncológico). Long-term follow-up of a randomized prospective trial comparing a standard 81 mg dose of intravesical bacille Calmette-Guerin with a reduced dose of $27 \mathrm{mg}$ in superficial bladder cancer. BJU Int. 2002;89(7):671-680.

24. Andius P, Holmang S. Bacillus Calmette-Guerin therapy in stage Ta/T1 bladder cancer: prognostic factors for time to recurrence and progression. BJU Int. 2004;93(7):980-984.

25. Herr HW, Badalament RA, Amato DA, Laudone VP, Fair WR, Whitmore WF Jr. Superficial bladder cancer treated with bacillus Calmette-Guerin: a multivariate analysis of factors affecting tumor progression. J Urol. 1989;141(1): 22-29.

26. Peyromaure M, Zerbib M. T1G3 transitional cell carcinoma of the bladder: recurrence, progression and survival. BJU Int. 2004;93(1):60-63.

27. Shahin O, Thalmann GN, Rentsch C, Mazzuchelli L, Studer UE. A retrospective analysis of 153 patients treated with or without intravesical bacillus Calmette-Guerin for primary stage $\mathrm{T} 1$ grade 3 bladder cancer: recurrence, progression and survival. J Urol. 2003;169(1):96-100.

28. Sylvester RJ, van der Meijden AP, Lamm DL. Intravesical bacillus Calmette-Guerin reduces the risk of progression in patients with superficial bladder cancer: a meta-analysis of the published results of randomized clinical trials. J Urol. 2002;168(5):1964-1970.

Dr. J. García Rodríguez

E-mail: jgrmed@hotmail.com

(Trabajo recibido el 9 de marzo 2006) 